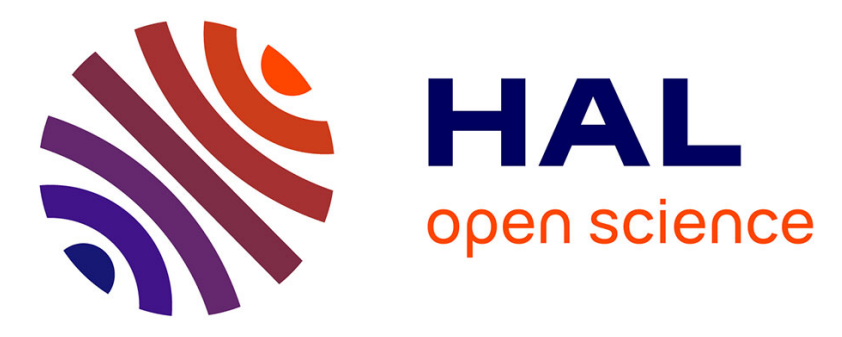

\title{
Interstitial 5-ALA photodynamic therapy and glioblastoma: preclinical model development and preliminary results.
}

Marie-Charlotte Tetard, Maximilien Vermandel, Henri-Arthur Leroy, Bertrand Leroux, Claude-Alain Maurage, Jean-Paul Lejeune, Serge Mordon, Nicolas

Reyns

\section{To cite this version:}

Marie-Charlotte Tetard, Maximilien Vermandel, Henri-Arthur Leroy, Bertrand Leroux, Claude-Alain Maurage, et al.. Interstitial 5-ALA photodynamic therapy and glioblastoma: preclinical model development and preliminary results.. Elsevier, 2015, pp.1/7. hal-01181127

\author{
HAL Id: hal-01181127 \\ https://hal.science/hal-01181127
}

Submitted on 25 Nov 2015

HAL is a multi-disciplinary open access archive for the deposit and dissemination of scientific research documents, whether they are published or not. The documents may come from teaching and research institutions in France or abroad, or from public or private research centers.
L'archive ouverte pluridisciplinaire HAL, est destinée au dépôt et à la diffusion de documents scientifiques de niveau recherche, publiés ou non, émanant des établissements d'enseignement et de recherche français ou étrangers, des laboratoires publics ou privés. 
Interstitial5-ALAphotodynamic therapy and glioblastoma: preclinical model development and preliminary results.

\author{
Marie-Charlotte Tetard, MD, MSc* \\ Maximilien Vermandel, $\mathrm{PhD}^{*}$ \\ Henri-Arthur Leroy, MD, MSc \\ Bertrand Leroux MSc, \\ Claude-Alain Maurage MD, $\mathrm{PhD}$, \\ Jean-Paul Lejeune MD, \\ Serge Mordon, PhD, \\ Nicolas Reyns, MD, PhD,
}

* These authors contributed equally to the paper

Corresponding author:

Maximilien Vermandel

m-vermandel@chru-lille.fr

Inserm U1189, ONCO-THAI

CHRU de Lille

1, avenue Oscar Lambret

59037, Lille - France 


\title{
Interstitial 5-ALA photodynamic therapy and glioblastoma: preclinical model development and preliminary results.
}

\author{
Marie-Charlotte Tetard ${ }^{1,2, \bullet}$, Maximilien Vermandel ${ }^{1,2^{*}, \bullet}$, Henri-Arthur Leroy ${ }^{1,2}$, \\ Bertrand Leroux ${ }^{1}$, Claude-Alain Maurage ${ }^{3}$, Jean-Paul Lejeune ${ }^{1,2}$, Serge Mordon ${ }^{1}$, Nicolas \\ Reyns ${ }^{1,2}$ \\ ${ }^{1}$ Univ. Lille, Inserm, CHU Lille, U1189 - ONCO-THAI - Image Assisted Laser Therapy for Oncology, F- \\ 59000 Lille, France \\ ${ }^{2}$ Department of Neurosurgery,CHU Lille, F-5900 Lille, France \\ ${ }^{3}$ Univ. Lille, CHU Lille, F-5900 Lille, France \\ * Corresponding author: m-vermandel@ chru-lille.fr \\ - These authors contributed equally to the paper
}

\begin{abstract}
:
Objective:Photodynamic therapy (PDT) has become a well-established modality for the treatment of many cancers. Photodynamic eradication of tumor cells depends on the presence of a photosensitizer, oxygen and light. However, oxygen depletion during PDT is awell knownproblem. Modulation of light deliverycould address this issue bycounteractingtumor hypoxia, therebyimproving tumor cell killing. This preclinical studywas designed to validate an animal model incorporating 5-aminolaevulinic acid(5-ALA)-PDT usingU87 glioblastoma cells. We aimed to evaluate the effects of light modulation for inducing specific tumoral lesions in this model (i.e., necrosis or apoptosis).
\end{abstract}

Materials and Methods: U87 glioblastoma cells were stereotactically engrafted intothe brains of male fox $1 \mathrm{rnu} / \mathrm{rnu}$ rats. Light delivery was studied after 5-ALA injection $(100 \mathrm{mg} / \mathrm{kg}$ i.p.). $26 \mathrm{~J}$ of $635 \mathrm{~nm}$ lightwas interstitially delivered to U87 tumor-bearing rats at a radiant power of either $30 \mathrm{~mW}$ (high fluence rate) or $4.8 \mathrm{~mW}$ (low fluence rate). In each group, half of the population received illumination in 2fractions with a refractory interval of 120 seconds, whereas the other half received continuous illumination.

Results:Twenty-two animals received 5-ALA-PDT, and the level of necrosis was scored. In the high-fluence-rate group, we observed a greater degree oftumor necrosis in rats receiving fractionated deliverythan inrats receiving continuous illumination. Similardifferenceswere not observed in the low-fluence-rate group, which exhibited only sparse necrosis.Higher morbidityand mortality rates wereobserved in the high-fluence-rate group.

Conclusion:We have developed a reproducible and reliable rodent model for interstitial 5ALA PDT. We found that the effects of 5-ALA-PDTare dependent on light delivery conditions. Although the low-fluence-rate treatment was bettertolerated, 5-ALA-PDT induced more necrosis using fractionated delivery at a high fluence rate.Theseresultsrequire 
confirmation with further studies involvinglarger populations and additional fractionation schemes.

Keywords: photodynamic therapy; high-grade glioma; 5-ALA; PpIX; U87;rodent model Conflict of interest: none 


\section{Introduction}

With an incidence of 3-5 cases/100,000 individuals/year, glioblastoma is the most common malignant primary tumor of the central nervous system $^{2}$.The standard treatment isa combination of surgery, radiotherapy and chemotherapy, andit achieves a median patient survival time of 15 months $^{37}$. The goal of surgery is to achieve maximum tumor cytoreduction while preserving neurological function ${ }^{42}$. The majority of treatment failures are due to local recurrence of the tumor, suggesting that more aggressive local therapy could be beneficial ${ }^{33}$. Photodynamic therapy (PDT) is a local treatment based on light activation of a photosensitizer (PS) using a laser of aspecific wavelength ${ }^{19,32}$. In the presence of oxygen, light interacts with the PS and formscytotoxic species. 5-Aminolevulinic acid (5-ALA) is a PS precursor. Indeed, the heme biosynthesis pathway leads to the production of 5-ALAinducedprotoporphyrin IX (PpIX), a endogenous PS ${ }^{32}$. PpIX is an excellent candidate for clinical PDT because of its highly selective uptake by tumors ${ }^{23,35,39}$. Furthermore, PpIX is relatively nontoxic, with a cutaneous photosensitization period limited to $1-2$ days $^{35,45}$. These qualities are exploited during Fluoro-Guided Resection (FGR) of high-grade gliomas using 5ALA.A randomized study demonstrated that complete resections of contrast-enhanced tumorswere more frequently achieved in malignant glioma patients when 5-ALA FGR was included $^{35}$.

Previously, clinical studies reported promising results when employing PDT on patients suffering from recurrences of high-grade gliomas, with a median survival increase from 39 months to 15 months ${ }^{4,34}$. Nevertheless, several issues still need to be addressed, including our ability toidentify the optimal doses of light and PS, the inter-individual variability in light distribution, and PS accumulation and tissue oxygenation. In particular, oxygen is a critical factor in the efficacy of PDT, as the cytotoxic effect of PDT is mediated by reactive oxygen species $^{17,32}$. Thus, in glioblastoma, pre-existing hypoxia is increased by the oxygen consumption required to carry out the photochemical reaction. This reversible tissue hypoxiamight significantly reduce the efficacy of PDT by decreasing the production of photochemical species ${ }^{12,17,21}$. Furthermore, the vasoconstriction of abnormal tumor microcirculation induced during PDT suppressesreperfusion andpotentially induces angiogenesis in the brain tissue adjacent to the tumor, subsequently contributing to tumor regrowth ${ }^{49}$.

Modulation of light delivery could decrease tissue hypoxia ${ }^{10,12}$, and low fluence rateshave beenreported to improve the efficiency of PDT, leading toincreasedapoptosis ${ }^{3,12,27}$. However, the delivery of such illumination schemes will likely require specific protocols or devices(e.g.,to repeatedly administer PS, an intracranial optical-fiber-based device might be necessary for several days).In addition, the application ofmultiple fractions of light exposure 12 at higher fluence ratesis being investigated as a means to counteract hypoxia.Fractions are defined as alternating 'on'/'off' periods that allow for reoxygenation Therefore, fractionation is expected to improve PDT because available molecular oxygencan be supplied by the capillary vasculature during the off-period.Fractionated PDT is promising because it may improve efficiency without dramatically increasing treatment time. Most studies on illuminationfractionation have investigated tumors in other locations, such as prostate cancers 
and skin lesions ${ }^{15,46}$. Studies ofhigh-grade gliomas failed todemonstrate any benefit of

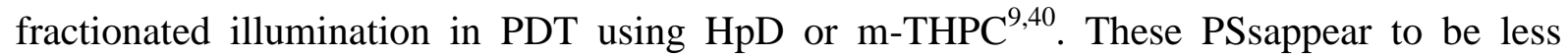
sensitive to fractionation, as they rapidly damaged tumor vasculature ${ }^{8}$. No experimental studies to date havereported the effects of fractionated 5-ALA-PDT on high-grade gliomas.

Based onpromising results inother areas of oncology, we investigated the effect of fractionated 5-ALA-PDT in a model of glioblastoma. Due to the importance of angiogenesis in glioblastoma, the most relevant modelinvolvesU87 gliomas engrafted into male fox 1 $\mathrm{rnu} / \mathrm{rnu}$ rat brains ${ }^{38}$. U87 is one of the most commonly used cell lines in the field of glioblastoma research ${ }^{6,18,22,25,48}$. This well-characterized cell line allows creates rapidly growing tumors with a high mitotic ratio and a satisfactory engraftment rate. Although U87 does not reproduce all the characteristics of human glioblastoma, such as spontaneous necrosis and an infiltrative pattern,these differences could be advantageous, as 1) the observed necrosis can be attributed completely to PDT, and 2) the absence of an infiltrative pattern should allow for more precise characterization of the treatment at a distance from the fiber tip.

Properly investigating the benefits of light fluence rates and of fractionation schemes requires a comprehensive experimental design including different groups of animals (e.g., high/low fluence rates, fractionation/no fractionation, andsham group with light/without light). Considering this, defining a preclinical model must be addressedbefore further studies can be performed. In this study, we define a proof of concept model as a basis for future studies. Thus, our main objective was to validate a preclinical model and experimental design for 5ALA PDTwith respect to treatment response, grafting procedure, histology analysis and imaging procedures. Second, we studied the influence of light fractionation and light fluence rate using this model to define preliminary trendsfor the treatment response. Toward this end, two fluence rates and one light fractionation scheme were evaluated to prove the utility of the procedures and outline protocols for further study.

\section{Materials and methods}

\subsection{Cell culture}

PDT experiments were conducted using the U87 human glioma cell line (ATCC HBT-14). The cell line was cryopreserved in liquid nitrogen. U87 cells were grown as monolayers in DMEM (Gibco Life Technologies, Grand Island, NY, USA) supplemented with $10 \%$ fetal bovine serum (FBS), penicillin, streptomycin, Glutamax, pyruvate, and essential and nonessential amino acids. Cell culture plates were incubated at $37^{\circ} \mathrm{C}$ and $5 \% \mathrm{CO}_{2}$. After harvesting the U87 cells with $0.25 \%$ trypsin, they were washed with Dulbecco's phosphatebuffered saline (PBS, Gibco Life Technologies) and counted.

\subsection{Experimental animals}

Athymic Fox1 rnu/rnu male rats (Harlan, Gannat, France) weighing 90-100 g (8 weeks old) at the start of the study were caged in air-filteredcages. The animal holding rooms were maintained at a constant temperature and humidity on a 12-hour light and dark schedule with an air exchange rate of 18 changes per hour. Animal care and protocols were in accordance with national legislation and institutional guidelines ${ }^{16,29}$.The rats were anesthetized with 
isoflurane (1-2\%) in oxygenduring tumor implantation, photo illumination and neuroimaging.

\subsection{5-ALA preparation and administration}

5-ALA was obtained as a hydrochloride powder. For intraperitoneal administration, 5-ALA was dissolved in PBS immediately before injection.All injections were intraperitoneal.

\subsection{Rat glioma model}

U87 glioblastoma cells $\left(5 \times 10^{5}\right.$ in $5 \mu \mathrm{l}$ ) were stereotactically implanted into the right putamenusing Paxinos atlas coordinates to induce intracerebral glioblastomas ${ }^{31}$. The anesthetized rats were fixed in a stereotactic frame (DavidKopf Instruments, Tujunga, CA, USA). The skin was incised under local anesthesia (lidocaine hydrochloride), and aburr hole was drilled at the following coordinates: $0.5 \mathrm{~mm}$ anterior and $2.7 \mathrm{~mm}$ to the right of the bregma. The needle was introduced through the burr hole and into the brain at a depth of $4.4 \mathrm{~mm}$, and $5 \mu \mathrm{l}$ of culture medium was injected. A delay of $10 \mathrm{~min}$ was observed between needle insertion into the brain and the onset of cell injection. The needle was left in place for $15 \mathrm{~min}$ after completingthe injection to prevent the spread of tumor cells during retraction. The cells were injected at a rate of $0.5 \mu 1 \cdot \mathrm{min}^{-1}$ via an $\mathrm{n}^{\mathrm{o}} 30-\mathrm{G}$ needle (RN G30 PST3 $51 \mathrm{~mm}$, Hamilton Company, Reno, NV, USA) fixed to a $10 \mu 1$ microsyringe (1700 model 1701-RN, Hamilton Company) controlled by an electrical pump (KDS 310; KD Scientific, Holliston, MA, USA). Prior to needle introduction, an anchor ${ }^{26}$ was erected to preserve a consistent position for grafting and treating. This anchor consisted of a cranial plate, a cannula projecting from the upper face of the plate and a prepositioning clip projecting from the bottom face of the plate. The cannula, the plate and the clip formed a hollow tube that guided the fiber perpendicular to the plate. The clip was positioned over the burr hole and then the anchor was positioned and fixed with biological tissue adhesive (Dermabond $\AA$, Johnson \& Johnson/Ethicon, Somerville, NJ, USA). Finally, closure was performed around the cannula of the anchor using Vicryl 4/0 (Ethicon, Somerville, NJ, USA).

\subsection{Magnetic resonance imaging (MRI)}

After engraftment, U87 tumor-bearing rats were followed-up periodicallyusing a small-animal 7T MRI system (BrukerBioSpec, Ettlingen, Germany). Followinganesthesia, animals were subjected toT2-weighted (T2W) fast spin echo pulse sequences $(\mathrm{TR}=5000 \mathrm{~ms}, \mathrm{TE}=77 \mathrm{~ms}$, slice thickness $=850 \mu \mathrm{m})$ and $\mathrm{T} 1$-weighted(T1W)fast spin echo pulse sequences $(\mathrm{TR}=400 \mathrm{~ms}$, $\mathrm{TE}=9 \mathrm{~ms}$, slice thickness $=850 \mu \mathrm{m}$ ) with and withoutgadolinium (T1WGd)-basedcontrast (MultiHence, $0.4 \mathrm{ml}$, i.p.). In the post-contrast studies, images were acquired 10 minutes after gadolinium administration. Tumor volumes were evaluated using standard viewing and reformatting software (OsiriX 4.1.2, 32-bit). The tumors were identifiedfrom their pathological contrast enhancement and the T2 signal. T2W sequences were alsolater used to assess perifocal edema. Tumor volume was computed by manual delineation based on each $\mathrm{T} 2 \mathrm{~W}$ image slice.The presence of an intratumoral hypointense signal was noted. MRI studies were performed at 7 and 14 days following engraftment and 48-72 hours after 5-ALA-PDT administration. 


\subsection{5-ALA-PDT of gross tumors}

To determine the effects of 5-ALA-PDT on the tumors, 22 animals were subjected to treatment 14days following inoculation with U87 cells. In all cases, animals received i.p.

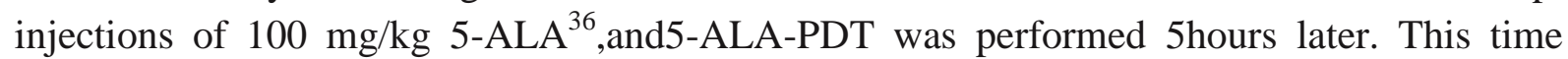
interval was chosen in accordance with previous experiments ${ }^{30,36}$.Prior to illumination, animals were anesthetized and positioned in the stereotactic frame. A $350-\mu \mathrm{m}$, bare, flat-end quartz fiber with a 0.29 numerical aperture was introduced through the anchor and into the brain at a depth of $4.4 \mathrm{~mm}$. The fiber was tightenedto the anchor, and the fiber was introduced into the tumor under the guidance of MRI.Light was delivered from a 635-nm diode laser. The animals were randomized into four treatment protocols. Animals in the continuous treatment modegroup were subjected to a radiant energy of $26 \mathrm{~J}$ at a radiant power of either $4.8 \mathrm{~mW}$ (low-fluence-rate group, $\mathrm{n}=5$ ) or $30 \mathrm{~mW}$ (high-fluence-rate group, $\mathrm{n}=6$ ). Forthe fractionated regime, rats were exposed to $26 \mathrm{~J}$ at $4.8 \mathrm{~mW}(\mathrm{n}=5)$ or $26 \mathrm{~J}$ at $30 \mathrm{~mW}(\mathrm{n}=6)$; in these two groups, the light dose was paused after $5 \mathrm{~J}$ for a duration of 120 seconds before delivering the remaining light dose $(21 \mathrm{~J})$. The rats were euthanized 72 hours after 5-ALA-PDT and subjected to a final MRI. Their brainswere thenremoved and prepared forhistopathology.

\subsection{Histological studies}

The rodent brains were removed and fixed in formaldehydefollowingsacrifice(48-72 hours after PDT) or premature death. Brains were cut in thecoronal plane (along the tumor injection trace) and subjected to HES staining.Tumor necrosis was scored by a senior neuropathologist who was blinded to the treatment modalitiesbased on the number of HE-stained sections throughout the tumorsas none, sparse, or extensive. Macrophagic invasion and apoptotic reactions were also recordedin the sections.

\section{Results}

Across all of the experiments, a tumor engraftment rate of $82 \%$ was achieved.

\subsection{Magnetic resonance imaging}

A total of 22animals were treated using interstitial PDT (iPDT). Within two days oftreatment, 7 of the animals died (Table 1). The remaining 15animals were investigated by MRI 48-72 hours after treatment. The tumors were observed on $\mathrm{T} 2 \mathrm{~W}$ as high-intensity signal areas at the sites of cell injection. No spread of tumor cells to other brain areas was detected. T2W images also revealededema in the brain adjacent to the tumor within a $2 \mathrm{~mm}$ radius. No necrotic or cystic regions were observed in these tumors.

The median tumor volume was $1.9 \mathrm{~mm}^{3}(0.35-5.1)$ on day 7 and $10 \mathrm{~mm}^{3}(0.84-55.2)$ on day 14following implantation. In particular, in terms of thefluence-rate groups, the median volume at 14 days was $15.8 \mathrm{~mm}^{3}\left(1.44-55.2 \mathrm{~mm}^{3}\right)$ in thehigh-fluence-rate group and $4.2 \mathrm{~mm}^{3}$ $\left(0.84-11.9 \mathrm{~mm}^{3}\right)$ in the low-fluence-rate group. Following 5-ALA-PDT, the median tumor volume was $19.3 \mathrm{~mm}^{3}\left(3.6-50.9 \mathrm{~mm}^{3}\right)$. For the post-treatment MRI, no significant 
changeswere visible using conventional sequences, including T2W and T1WGd images.

Table 1. Assignment of animals to various illumination groups.

\subsection{Histology: variations in fluence rateandfractionation of light delivery}

In total, 22 animals subjected to treatment were analyzed. Sevenanimalsfrom the highfluence-rate group died within 24 hours of treatment (4 in the fractionated group and 3inthecontinuous group). The remaining 15 animals were sacrificed following post-treatment MRI at 48-72 h. Representative sections from the treated tumors are presented in Fig. 1, where the fiber tip path is shown(path estimated according to MRI slice, Fig. 1 (a)) on the internal margin of the tumor.

Fig 1. Histological sections

(a) Fiber path viewed by MRI.(b) Low-magnification (x10) microphotographs show sparse necrosis with an intact tumor;fiber path is indicatedwith a dashed arrow. (c) Extensive tumor necrosis. (d) High-magnification microphotographs (x20) showing the tumor border in a normal brain $(B)$, necrosis $(N)$, and an intact tumor core $(T)$. (e) Necrosis and apoptotic bodies at higher magnification.

Thelevels of 5-ALA-PDT-induced necrosis in the HES-stained sections are shown in Table 2.Necrosis was observedinboththe high- and low-fluence-rate groups. In most cases, the necrosis was sparse andlocalized around the fiber tip. Inthe high-fluence-rate group, increased necrosis was observed afterfractionated illuminationcompared with the continuous illumination group. Bycontrast, necrosis wassparse in the low-fluence-rate group even after fractionated treatment.

Table 2. Tumor necrosis in HE-stained sections after 5-ALA-PDT treatment with $26 \mathrm{~J}$ of light delivered at high or low fluence rates with fractionated or continuous illumination.

Macrophage infiltration and apoptotic reactions were also observed in the necrotic and intact tumor areas, and no 5-ALA-PDT-induced necrosis was detected in the normal brain. However, edema was observed in the brain tissue adjacent to the tumor, particularly after high-fluence rate illumination.

Fig 2. Edema observed on T2w MRI.

\subsection{Morbidity of interstitial PDT}

Interstitial 5-ALA-PDT delivered at a high fluence rate 14 days after tumor induction resulted in clinical signs of elevated Intra Cranial Pressure (ICP) and wasfatal in 58\% (7/12) of the animals in these groups. Over the course of our experiments, corticoids were used to counteract unexpected animal death when applying high fluence rates. Consequently, 4 of these 7 rats were not administered methylprednisolone following treatment. Therefore, the 
steroids were expectedto increase treatment tolerance.

All animals receiving 5-ALA-PDT at a low fluence rate survived the treatment and did not exhibit the severe symptoms of increased ICP after light exposure.

Theobserved post-treatment morbidity resulted froma combination of the 5-ALA-PDT response, the tumor volume and,most likely, hydrocephalus. Indeed, MRI images indicated several cases of hydrocephalus related to trapping of the ipsilateral horn of the lateral ventricle,which could have increased ICP. Two animals with hematomascaused by introduction of the optical fiber were also noted; one resulted in the death of the animal. Concerning the administration of 5-ALA, no adverse effect was observed.

\section{Discussion}

Based on our results, 5-ALA-induced PpIX appears to be a valuable PSbased on several criteria. First,good clinical tolerance was observed, ${ }^{35}$ andprevious experimental and clinical studies demonstrated a high degree of selectivity in malignant glioma tissues ${ }^{3,23,36,41}$

The main factor limiting the efficacy of 5-ALA-PDT in the treatment of malignant glioma is tumor hypoxia, either pre-existing in the tumor or as a result of oxygen depletion during PDT. This hypoxia limits photochemical reactions that depend on oxygenand can consequently cause cellular destruction. Moreover, as a side effect, tumor hypoxia can induce angiogenesis and potentiate tumor regrowth.

In our study, we first aimed to validate a preclinical model and experimental protocol for 5ALA PDT with respect to treatment response, grafting procedure, histology analysis and imaging procedures. These preliminary experiments enabled us to evaluate the effects of light modulation in this system. Indeed,previous studies suggested that the modulation of light delivery could enhance the efficiency of 5-ALA-PDT ${ }^{3,5,20,27}$. For a given total light dose, low fluence rates were reported to be more effective compared with higher rates, ${ }^{3,5,27}$ most likely because stronger and faster $\mathrm{O}_{2}$ depletion wasassociated with higher fluence rates ${ }^{12}$. In our experiments, necrosis was observed at both low and high fluence rates. Nevertheless, the efficiency of the high fluence rate conflicted withprior results reported in the literature ${ }^{3,5,30}$.It is possible that the type of tumor used in our experiments could explain this discrepancy, asU87 tumorsare homogeneous, hypervascularized,and lack necrosis ${ }^{7}$. Furthermore, we observed alarge rangeof tumor volumes, which is a significant drawback of this investigation. In addition to randomizing the animals, this issue could have been avoided by waiting until MRI showed a minimum preset volume.In addition to necrosis, the neuropathologist also qualitativelyobserved macrophage infiltration and apoptotic reactions in the necrotic and intact tumor areas, although this observation requires quantitative investigation. Future studies involving specific apoptosis analyses should be performed using immunohistochemistry with the TUNEL method (Apoptag ${ }^{\circledR}$ Plus Peroxidase In Situ, Millipore, USA).

The second part of our experimental work was designed to assess the enhanced efficacy of 5ALA-PDT using light fractionation. The number of tumor-bearing animals available in this 
preliminary studywas limited, but the data strongly indicated that lightfractionation enhanced the effect of 5-ALA-PDT on tumor tissue. However, the effect of light fractionation was critically dependent on the treatment conditions. At the high fluence rate, our experiment revealed that the area of PDT necrosis increased when a single pause was introduced after delivering $5 \mathrm{~J}$ compared with continuous illumination. Although we did not find a similar difference at the low fluence rate, our results generally agreed with those of previous studies $^{11,14,15}$, whose authors postulated three processes to explain the efficacy of fractionated PDT. First, light exposure initially caused temporary vascular occlusion followed by hypoxia when the consumption of oxygen surpassesthe flow supplied by abnormal blood vessels. The dark period enabled reoxygenation of the tumor, making the subsequent light deliverymore efficient. The two other processes involve reperfusion injury and relocalization of the PS during the dark interval. Some studies have suggested that the effects are lessened when a low fluence rate is applied because the consumption of oxygen is slower ${ }^{3,5,20}$. Moreover, the benefits of fractionation was shown to be greater for 5-ALA than for certain other photosensitizers, based on its intracellular effect ${ }^{14,43}$.PDT withmTHPC or Photofrin ${ }^{\circledR}$ (which exhibits a vascular effect)as the PS was not enhanced by fractionation of the light delivery 9,40 .

In our study, we used a fractionation scheme described by Curnow et al. in the normal rat colon in which 5of the total $25 \mathrm{~J}$ was delivered in the first fraction ${ }^{14}$. Curnow et al.demonstrated the importance of timingthe light delivery before the dark periodto avoid destroying the microcirculation necessary for further reoxygenation ${ }^{13}$. Another important feature ofthe dark period duration is thatthe $\mathrm{O}_{2}$ recovery phase is likely to be tumor-type dependent owing to variations in vessel density, flow rate and oxygenation.It has therefore proven difficult thus far to define the optimal fractionation scheme. This change in oxygenation could potentially be monitored by measuringthe partial pressure of oxygen in the glioma and adjusting the duration of the on/off sequences ${ }^{24}$.

Clinical tolerancewas another factor affectingPDT efficiency. The observed post-treatment morbidity was likely due to increased ICP. These results suggested that a low fluence rate was better tolerated;however, it should be noted that, despite randomization, the average tumor volume in the high-fluence-rate group was higher than that in the low-fluence-rate group: 15.8 $\mathrm{mm}^{3}$ (1.44-55.2) versus $4.2 \mathrm{~mm}^{3}$ (0.84-11.9), respectively. Thus, PDT-induced edema contributed to the ICP and,in some cases, caused mortality when the tumor volume was $\operatorname{high}^{4,28}$.

This 5-ALA-PDT-induced edema is a common complication following PDT of brain tumors. It is typically addressed by administering steroids that improve clinical tolerance ${ }^{3,4,28}$. However, in our experiments, the effects of this adjuvant treatment were impaired when treating a high-volume tumor. Furthermore, tumor volume isknown to be a limiting factor during interstitial treatment ${ }^{4}$.

MRI is the most important tool for assessing the management of cerebral gliomas, although in our study,PDT efficacy was not properly evaluated usingT1W, T1WGd and T2W sequences. These results are not consistent with those reported by Beck et al. in a clinical study, which 
demonstrated a partial or complete resolution of tumorcontrast enhancement within 24 hours after 5-ALA-PDT ${ }^{4}$.Based on these preliminary experiments, we did not find any MRI evidence to corroborate our pathological findings. However, some groupshave reported the use of diffusion-weighted and perfusion MRIto evaluate the effects of vascular-targeted interstitial $\mathrm{PDT}^{1,44}$.Therefore, the use of multiparametric MRI toassess PDT efficacy,without the need for histological examination,should be validated with comprehensive experimental designs, including low and high fluence rates, fractionation and continuous lighting assessed by sophisticated pathological analyses (e.g., apoptosis and necrosis).

Finally, the dose of $100 \mathrm{mg} / \mathrm{kg}$ used in this study was based on a former study by Stummer et al. ${ }^{36}$. The optimal dose has to be evaluated to optimize the PDT effects. A fluorescence microscopy study might be achieved for different doses to assess the optimal dose level.

\section{Conclusion}

In this study, we report the use ofinterstitial 5-ALA-PDT in a U87 glioblastoma rodent model. As angiogenesis is generally associated with glioblastoma, rats bearing U87 tumors rather than C6 tumors were chosen for these experiments ${ }^{36,47}$.We established a reproducible and reliable rodent model for 5-ALA interstitial PDT. This new approach towardinvestigating 5ALA-PDTallowed us to observe that its effect was critically dependent on the light delivery conditions. We therefore focused this study on assessing 1) the effects of interstitial PDT at high versus low fluence ratesof delivery and 2) the effect of fractionated light delivery. Our results suggest that fractionated PDT was more effective compared with continuous light delivery.Furthermore, we noted that the efficacy of fractionated delivery also depended on the rate of light delivery, with stronger effectsatthe high fluence rate. Indeed, fractionated light exposure at a radiant power of $30 \mathrm{~mW}$ induced more necrosis compared with that observed at $4.8 \mathrm{~mW}$ (either by fractionated or continuous PDT). However, low-fluence-rate 5-ALA-PDT was stillableto induce significant tumor necrosis.Finally, we show proof-of-concept experiments using the U87 glioblastoma rodent model to assess the effects of PDT. Future experiments should be performed to validate the effect of fluence rate and fractionation on pathology and to identify MRI markers forthese treatment effects. Toward this end, we are currently testing a significantly larger group ofanimals receiving $25 \mathrm{~J}$ at a rate of $30 \mathrm{~mW}$ using two different fractionation schemes (compared with asham group) to evaluatemorbidity and side effects. Specific apoptosis analyses using immunohistochemistry with the TUNEL methodare also planned.

Grants: The French Society of Neurosurgery and the French Institute of Health and Medical Research (INSERM) supported this work.

Acknowledgments: The authors warmly thank Florent Auger and Myriem Tardivel for their involvement in the study. 
1. Afaq A, Koh DM, Padhani A, van As N, Sohaib SA: Clinical utility of diffusionweighted magnetic resonance imaging in prostate cancer. BJU Int 108:17161722, 2011

2. Ahmed R, Oborski MJ, Hwang M, Lieberman FS, Mountz JM: Malignant gliomas: current perspectives in diagnosis, treatment, and early response assessment using advanced quantitative imaging methods. Cancer Manag Res 6:149-170, 2014

3. Angell-Petersen E, Spetalen S, Madsen SJ, Sun CH, Peng Q, Carper SW, et al: Influence of light fluence rate on the effects of photodynamic therapy in an orthotopic rat glioma model. J Neurosurg 104:109-117, 2006

4. Beck TJ, Kreth FW, Beyer W, Mehrkens JH, Obermeier A, Stepp H, et al: Interstitial photodynamic therapy of nonresectable malignant glioma recurrences using 5aminolevulinic acid induced protoporphyrin IX. Lasers Surg Med 39:386-393, 2007

5. Bisland SK, Lilge L, Lin A, Rusnov R, Wilson BC: Metronomic photodynamic therapy as a new paradigm for photodynamic therapy: rationale and preclinical evaluation of technical feasibility for treating malignant brain tumors. Photochem Photobiol 80:22-30, 2004

6. Burckel H, Josset E, Denis JM, Gueulette J, Slabbert J, Noel G, et al: Combination of the mTOR inhibitor RAD001 with temozolomide and radiation effectively inhibits the growth of glioblastoma cells in culture. Oncol Rep, 2014

7. Candolfi M, Curtin JF, Nichols WS, Muhammad AG, King GD, Pluhar GE, et al: Intracranial glioblastoma models in preclinical neuro-oncology: neuropathological characterization and tumor progression. J Neurooncol 85:133-148, 2007

8. Castano AP: Mechanisms in photodynamic therapy: Part three-Photosensitizer pharmacokinetics, biodistribution, tumor localization and modes of tumor destruction. Photodiagnosis and Photodynamic Therapy:91-106, 2005

9. Chen Q, Chopp M, Dereski MO, Wilson BC, Patterson MS, Schreiber A, et al: The effect of light fluence rate in photodynamic therapy of normal rat brain. Radiat Res 132:120-123, 1992

10. Chen Q, Huang Z, Chen H, Shapiro H, Beckers J, Hetzel FW: Improvement of tumor response by manipulation of tumor oxygenation during photodynamic therapy. Photochem Photobiol 76:197-203, 2002

11. Curnow A, Bown SG: The role of reperfusion injury in photodynamic therapy with 5-aminolaevulinic acid--a study on normal rat colon. Br J Cancer 86:989-992, 2002

12. Curnow A, Haller JC, Bown SG: Oxygen monitoring during 5-aminolaevulinic acid induced photodynamic therapy in normal rat colon. Comparison of continuous and fractionated light regimes. J Photochem Photobiol B 58:149-155, 2000

13. Curnow A, MacRobert AJ, Bown SG: Comparing and combining light dose fractionation and iron chelation to enhance experimental photodynamic therapy with aminolevulinic acid. Lasers Surg Med 38:325-331, 2006

14. Curnow A, McIlroy BW, Postle-Hacon MJ, MacRobert AJ, Bown SG: Light dose fractionation to enhance photodynamic therapy using 5-aminolevulinic acid in the normal rat colon. Photochem Photobiol 69:71-76, 1999

15. De Bruijn HS, van der Ploeg-van den Heuvel A, Sterenborg HJ, Robinson DJ: Fractionated illumination after topical application of 5-aminolevulinic acid on 
normal skin of hairless mice: the influence of the dark interval. J Photochem Photobiol B 85:184-190, 2006

16. DIRECTIVE 2010/63/EU OF THE EUROPEAN PARLIAMENT AND OF THE COUNCIL of 22 September 2010 on the protection of animals used for scientific purposes, THE EUROPEAN PARLIAMENT, 2010

17. Foster TH, Murant RS, Bryant RG, Knox RS, Gibson SL, Hilf R: Oxygen consumption and diffusion effects in photodynamic therapy. Radiat Res 126:296-303, 1991

18. Han N, Shahveranov A, Cheng Y, Qin K, Yu SY, Zhang MX: Effects of connective tissue growth factor (CTGF) gene silencing on the radiosensitivity of glioblastoma. Int J Clin Exp Med 7:2557-2563, 2014

19. Henderson BW, Dougherty TJ: How does photodynamic therapy work? Photochem Photobiol 55:145-157, 1992

20. Henderson BW, Gollnick SO, Snyder JW, Busch TM, Kousis PC, Cheney RT, et al: Choice of oxygen-conserving treatment regimen determines the inflammatory response and outcome of photodynamic therapy of tumors. Cancer Res 64:21202126, 2004

21. Henning JP, Fournier RL, Hampton JA: A transient mathematical model of oxygen depletion during photodynamic therapy. Radiat Res 142:221-226, 1995

22. Jarzabek MA, Amberger-Murphy V, Callanan JJ, Gao C, Zagozdzon AM, Shiels L, et al: Interrogation of gossypol therapy in glioblastoma implementing cell line and patient-derived tumour models. Br J Cancer, 2014

23. Johansson A, Palte G, Schnell O, Tonn JC, Herms J, Stepp H: 5-Aminolevulinic acidinduced protoporphyrin IX levels in tissue of human malignant brain tumors. Photochem Photobiol 86:1373-1378, 2010

24. Khan N, Mupparaju S, Hou H, Williams BB, Swartz H: Repeated assessment of orthotopic glioma $\mathrm{pO}(2)$ by multi-site EPR oximetry: a technique with the potential to guide therapeutic optimization by repeated measurements of oxygen.

J Neurosci Methods 204:111-117, 2011

25. Marrero L, Wyczechowska D, Musto AE, Wilk A, Vashistha H, Zapata A, et al: Therapeutic efficacy of aldoxorubicin in an intracranial xenograft mouse model of human glioblastoma. Neoplasia 16:874-882, 2014

26. Marty E, Mordon S, Bechet D, Barberi-Heyob MA: Cranial anchor, in, ed A61M 25/02 (2006.01); A61B 19/00 (2006.01); A61N 1/05 (2006.01); A61M 39/02 (2006.01), 2012

27. Mathews MS, Angell-Petersen E, Sanchez R, Sun CH, Vo V, Hirschberg H, et al: The effects of ultra low fluence rate single and repetitive photodynamic therapy on glioma spheroids. Lasers Surg Med 41:578-584, 2009

28. Mathews MS, Chighvinadze D, Gach HM, Uzal FA, Madsen SJ, Hirschberg H: Cerebral edema following photodynamic therapy using endogenous and exogenous photosensitizers in normal brain. Lasers Surg Med 43:892-900, 2011

29. National charter on the ethics of animal experimentation, French ministry of higher education and research, 2008

30. Olzowy B, Hundt CS, Stocker S, Bise K, Reulen HJ, Stummer W: Photoirradiation therapy of experimental malignant glioma with 5-aminolevulinic acid. J Neurosurg 97:970-976, 2002

31. Paxinos G, Watson $\mathrm{C}$ : The rat brain in stereotaxic coordinates, ed 6th. Amsterdam ; Boston ;: Academic Press/Elsevier, 2007 
32. Peng Q, Warloe T, Berg K, Moan J, Kongshaug M, Giercksky KE, et al: 5Aminolevulinic acid-based photodynamic therapy. Clinical research and future challenges. Cancer 79:2282-2308, 1997

33. Petrecca K, Guiot MC, Panet-Raymond V, Souhami L: Failure pattern following complete resection plus radiotherapy and temozolomide is at the resection margin in patients with glioblastoma. J Neurooncol 111:19-23, 2013

34. Stummer W, Beck T, Beyer W, Mehrkens JH, Obermeier A, Etminan N, et al: Longsustaining response in a patient with non-resectable, distant recurrence of glioblastoma multiforme treated by interstitial photodynamic therapy using 5ALA: case report. J Neurooncol 87:103-109, 2008

35. Stummer W, Pichlmeier U, Meinel T, Wiestler OD, Zanella F, Reulen HJ: Fluorescence-guided surgery with 5-aminolevulinic acid for resection of malignant glioma: a randomised controlled multicentre phase III trial. Lancet Oncol 7:392-401, 2006

36. Stummer W, Stocker S, Novotny A, Heimann A, Sauer O, Kempski O, et al: In vitro and in vivo porphyrin accumulation by C6 glioma cells after exposure to 5aminolevulinic acid. J Photochem Photobiol B 45:160-169, 1998

37. Stupp R, Mason WP, van den Bent MJ, Weller M, Fisher B, Taphoorn MJ, et al: Radiotherapy plus concomitant and adjuvant temozolomide for glioblastoma. $\mathbf{N}$ Engl J Med 352:987-996, 2005

38. Tetard MC, Vermandel M, Mordon S, Lejeune JP, Reyns N: Experimental use of Photodynamic Therapy in high grade gliomas: a review focused on 5aminolevulinic acid. Photodiagnosis Photodyn Ther 11:319-330, 2014

39. Tetard MC, Vermandel M, Mordon S, Lejeune JP, Reyns N: Experimental use of Photodynamic Therapy in high grade gliomas: a review focused on 5aminolevulinic acid. Photodiagnosis Photodyn Ther, 2014

40. Tudge SH, Kaye AH, Hill JS: Modulation of light delivery in photodynamic therapy of brain tumours. J Clin Neurosci 6:227-232, 1999

41. Utsuki S, Oka H, Sato S, Shimizu S, Suzuki S, Tanizaki Y, et al: Histological examination of false positive tissue resection using 5-aminolevulinic acidinduced fluorescence guidance. Neurol Med Chir (Tokyo) 47:210-213; discussion 213-214, 2007

42. Van den Bent MJ, Stupp R, Mason W, Mirimanoff RO, Lacombe D, Gorlia T: Impact of the extent of resection on overall survival in newly-diagnosed glioblastoma afer chemo-irradiation with temozolamide: further analysis of EORTC study 26981. Eur J Cancer Suppl. 3:134, 2005

43. Van der Veen N, van Leengoed HL, Star WM: In vivo fluorescence kinetics and photodynamic therapy using 5-aminolaevulinic acid-induced porphyrin: increased damage after multiple irradiations. Br J Cancer 70:867-872, 1994

44. Wang H, Fei B: Diffusion-weighted MRI for monitoring tumor response to photodynamic therapy. J Magn Reson Imaging 32:409-417, 2010

45. Webber J, Kessel D, Fromm D: Side effects and photosensitization of human tissues after aminolevulinic acid. J Surg Res 68:31-37, 1997

46. Xiao Z, Halls S, Dickey D, Tulip J, Moore RB: Fractionated versus standard continuous light delivery in interstitial photodynamic therapy of dunning prostate carcinomas. Clin Cancer Res 13:7496-7505, 2007

47. Zelenkov P, Baumgartner R, Bise K, Heide M, Meier R, Stocker S, et al: Acute morphological sequelae of photodynamic therapy with 5-aminolevulinic acid in the C6 spheroid model. J Neurooncol 82:49-60, 2007 
48. Zhang J, Zhou Q, Gao G, Wang Y, Fang Z, Li G, et al: The effects of ponatinib, a multi-targeted tyrosine kinase inhibitor, against human U87 malignant glioblastoma cells. Onco Targets Ther 7:2013-2019, 2014

49. Zhang X, Jiang F, Zhang ZG, Kalkanis SN, Hong X, deCarvalho AC, et al: Low-dose photodynamic therapy increases endothelial cell proliferation and VEGF expression in nude mice brain. Lasers Med Sci 20:74-79, 2005 